\title{
Influencia de estrategias metacognitivas en logros académicos del área Personal Social, V ciclo, de una Institución Educativa de Trujillo, 2020.
}

\author{
Marco Antonio Escudero Saldarriaga \\ mescuderos@ucvvirtual.edu.pe \\ Universidad Cesar Vallejo - Perú \\ Dulio Oseda Gago \\ doseda@ucvirtual.edu.pe \\ https://orcid.org/0000-0003-3136-6094 \\ Universidad Cesar Vallejo - Perú
}

\section{RESUMEN}

El presente trabajo tuvo como objetivo determinar la influencia de las estrategias metacognitivas en los logros académicos en el área de Personal Social en V ciclo de una institución educativa de Trujillo, 2020, para lo cual se utilizó una investigación de tipo básica; el diseño de la investigación es no experimental, transversal, correlacional causal, con una población de 155 Estudiantes y una muestra de 95 seleccionados por conveniencia no aleatoria, a quienes se les administró dos cuestionarios como instrumentos para el recojo de información relacionada con las variables. Los resultados confirman la hipótesis principal sobre la influencia de las estrategias metacognitivas en los logros académicos del área de Personal Social en un 77,5 \% y una relación directa y fuerte (Rho Spearman $r=0,804 * *$ y $p<0.005$ ) por lo que se concluye afirmando que cuando los estudiantes usan estrategias metacognitivas, esto repercute en la mejora de los logros académicos.

Palabras clave: Cognición, Metacognición, Logros académicos. 
The objective of this work was to determine the influence of metacognitive strategies on academic achievements in the area of Social Personnel in $\mathrm{V}$ cycle of an educational institution in Trujillo, 2020

\begin{abstract}
The objective of this work was to determine the influence of metacognitive strategies on academic achievements in the area of Social Personnel in V cycle of an educational institution in Trujillo, 2020, for which a basic type investigation was used; The research design is non-experimental, cross-sectional, causal correlation, with a population of 155 Students and a sample of 95 selected for non-random convenience, who were administered two questionnaires as instruments for collecting information related to the variables The results confirm the main hypothesis about the influence of metacognitive strategies on academic achievements in the area of Social Personnel in $77.5 \%$ and a direct and strong relationship (Rho Spearman $r=0.804 * *$ and $p<0.005$ ), therefore concludes stating that when students use metacognitive strategies, this affects the improvement of academic achievement.
\end{abstract}

Keywords: Cognition, Metacognition, Academic achievements.

Artículo recibido: 10. Mayo. 2021 Aceptado para publicación: 28. junio. 2021 Correspondencia: marcoescuderosaldarriaga@gmail.com Conflictos de Interés: Ninguna que declarar 


\section{INTRODUCCIÓN}

Frente a un panorama adverso de rendimiento académico, es que las estrategias en el aprendizaje resultan ser fundamentales en la obtención del éxito de los objetivos académicos propuestos, en efecto, las investigaciones en los últimos años han cambiado nuestras percepciones respecto de los roles del binomio alumno y docente; tener un aprendizaje independiente y autorregulado, por lo que es importante introducir las denominadas estrategias metacognitivas en las aulas de clase, como apoyo para los estudiantes que en su proceso de aprendizaje presenten problemas que deban superar para el logro de los fines propuestos.

Las estrategias metacognitivas son herramientas que ayudan al aprendizaje del alumno, tal como señala Jaramillo y Simbaña (2014), que son formas de aprendizaje que busca en los estudiantes el “ aprender a aprender" formando en ellos autoconciencia y autorregulación, a través de estrategias metacognitivas teórico-prácticas posibles de aplicar en la práctica docente; asimismo incorpora en los procesos psicopedagógicos los entornos virtuales que potencian la comprensión del conocimiento. (pág. 312). "Los estudiantes que interactuaron con el andamiaje metacognitivo mostraron un mayor logro de aprendizaje y una mejor capacidad de monitoreo de su proceso de aprendizaje". Solórzano y López (2019)

La institución educativa 81758 “Telmo Hoyle de Los Ríos”, localizada en el populoso y pujante Distrito de Huanchaco, Provincia de Trujillo, Departamento de La Libertad, perteneciente a la UGEL 03 Trujillo Nor Oeste, no es ajena a esta problemática, tal como lo señala el MINEDU (2018), presenta un $7.6 \%$ de previo al inicio de la comprensión de lectura, $34.3 \%$ en inicio, el $36.8 \%$ en proceso y $21.3 \%$ satisfactorio, es decir, casi el $79 \%$ demuestra falencias en la comprensión lectora, situación que impone como necesario la incorporación de estrategias de aprendizaje para potencializar el proceso del conocimiento.

Bajo esta realidad problemática, se hace necesario que el Estado Peruano impulse políticas adecuadas para mejorar en sus diferentes niveles el aprendizaje del estudiantado peruano, por tanto, el presente trabajo plantea como problema de estudio: ¿Cuál es la influencia de la estrategia metacognitiva en los logros académicos del área Personal Social, en estudiantes de V ciclo de una Institución Educativa de Trujillo 2020? Este problema así planteado refleja no sólo nuestra preocupación respecto de esta realidad y 
problemática educativa en nuestro distrito, sino además a través del mismo se busca transparenta el compromiso asumido con el quehacer educativo en nuestra localidad, para lograr no sólo mejorar las destrezas y obtener mayor cantidad de conocimientos en nuestros educandos, sino principalmente formar personas con capacidad reflexiva y por ende mejores seres humanos.

Se justifica el trabajo según su conveniencia, por cuanto se analizarán las posiciones de los diferentes autores, sobre la influencia de la estrategia metacognitiva en los logros académicos del área Personal Social, en la institución educativa del estudio. Con ello podremos abordar las diferentes aristas de esta problemática educativa y con ello aportar con nuestro trabajo a mejorar el proceso de enseñanza - aprendizaje en nuestra localidad. El objetivo general es: Determinar la influencia que tiene la estrategia metacognitiva en los logros académicos del área Personal Social, en estudiantes de V ciclo en la Institución Educativa $N^{\circ} 81758$ Trujillo 2020. Como se aprecia el objetivo general está redactado en forma clara y precisa y guarda perfecta relación con lo que se pretende investigar.

En el contexto nacional, Cansaya (2018) en la tesis que titulara "Las estrategias metacognitivas y el aprendizaje significativo en el área de arte, colegio Emblemático del Perú Mateo Pumacahua, Cuzco, año 2015”, realizada en la Universidad Nacional de Educación Enrique Guzmán Valle determinó en sus conclusiones que existe evidente relación o en otros términos una relación en grado significativo entre las estrategias metacognitivas con el aprendizaje de los educandos.

Anaya (2018), en su tesis titulada "Aplicación de estrategias cognitivas y metacognitivas y su influencia en la comprensión lectora en primaria Mirones Lima - 2017”, realizada en la Universidad Nacional Federico Villarreal, determinó en sus conclusiones que aplicando las estrategias cognitivas y metacognitivas en la comprensión lectora de los educandos del quinto año A del nivel primario en la Institución educativa Nuestra Señora del Carmen de Mirones, Lima. 2017, se obtuvieron una destacada comprensión lectora, siendo de destacar que aquellos estudiantes que se encontraban en un nivel previsto subieron al nivel destacado.

Laguna y Trujillo (2017), referenciado a Gonzales (2003) señalan las características de las estrategias de aprendizaje las cuales permiten que el alumno aprenda a formular sus interrogantes; permiten que el estudiante planifique sus estrategias, en el afán de aprender; facilitan analizar los factores que le favorecen o dificultan el aprender; conocer 
procedimientos para ver sus resultados; se utilizan métodos y procesos para la revisión de las tareas realizadas, pudiendo reformular sus objetivos.

En esa misma línea, Córdova y Marroquín (2018) mencionan "que las estrategias metacognitivas para el aprendizaje significativo mejoraron el rendimiento académico de los estudiantes", de allí la importancia del presente estudio que permite corroborar los resultados obtenidos por los investigadores, en la importancia del uso de estrategias metacognitivas en los estudiantes. De igual forma en cuanto a la estrategia cognitiva Cabero, et al. (2017) concluyen que el proceso de aprendizaje está relacionado directamente con el tipo de estrategias y con la madurez del sujete, entre ellas la metacognitivas. Por otro lado, a decir de Chablé (2020) afirma que la comprensión lectora, tiene una relación directa con el uso de ciertas estrategias siendo las más resaltantes las metacognitivas, por cuanto permite una reflexión continua del lector, al interactuar con los textos. De igual Muhid, et al. (2020) cuyos resultados indicaron que "las estrategias metacognitivas tuvieron un efecto positivo en el rendimiento en lectura de los estudiantes".

Respecto de las estrategias metacognitivas Osses y Jaramillo (2008) establece que viene a ser el grupo de acciones que se orientan al conocimiento de los procesos mentales y las operaciones, para con ello saber utilizarlas, readaptarlas y también cambiarlas cuando sea necesaria en relación con las metas propuestas y encaminadas. (pág. 193); del mismo modo Alvarado y Lunarejo (2017), la define "como saberes sobre los procesos de cognición o autoadministración de estudios, mediante la planeación, monitoreo y evaluación" (pág. 38); por otro lado, Cansaya (2018), las define como aquel grado de conocimiento que tienen los sujetos y también su grado de conciencia en relación al proceso de pensar, a los contenidos que engloba las estructuras, las habilidades tendientes a organizarlos, modificarlos y revisarlos en forma periódica relacionados con el proceso de aprendizaje. (pág. 35). Susantini y otros (2018) concluyen que "los estudiantes de alta capacidad realizaron mayores habilidades metacognitivas"; y por otro lado Lestari y Jailani (2018) determinan que "Los estudiantes que están expuestos a las estrategias metacognitivas superan significativamente a sus contrapartes" Analizadas estos resultados y definiciones, se tiene que el estudiante debe autorregular sus procesos de la mente y sus cogniciones, así como también su percepción, comprensión entre otros aspectos, con el fin de saber, en qué momento usarlas, para resolver las tareas que se les 
presentan, del mismo modo estas estrategias pueden ser reajustadas cuando así lo requiere el estudio.

La pedagogía en las estrategias de aprendizaje es muy importante en el conocimiento del alumno, tal como lo precisa Sánchez, et al. (2017), Los estudiantes de nivel superior superaron significativamente a los estudiantes de nivel inferior en orientaciones metacognitivas, así lo menciona Ajaja (2017) Rogers, (2018) en sus resultados del estudio indicaron que los estudiantes emplearon más estrategias cognitivas y menos estrategias metacognitivas. Al señalar que para desarrollar y lograr el proceso aprendizaje docente y de enseñanza, se debe contar con una variedad de instrumentos que le permitan llegar e interactuar con el estudiante, permitiendo con ello la motivación por el tema, el cual finalmente debe llegar al alumno de manera significativa; para ello el docente debe estar preparado, en razón de ello, el diseño de estrategias debe orientarse a propósitos específicos y de acuerdo a la necesidades y características del grupo, lo cual conlleva a obtener resultados óptimos y satisfactorios, por tanto se hace necesario que el docente incorpore procesos básicos que permitan llegar con claridad al alumno, en otras palabras debe tener la capacidad de enseñar a otro a aprender de por vida; del mismo modo según Chia, et al. (2019) señalan en sus resultados que, "las estrategias propuestas ayudaron a mejorar la conciencia metacognitiva de los estudiantes", asimismo Sierra (Sierra, 2007), indica además que los concepto teóricos y prácticos en el proceso de enseñanza que lleva transformar de un estado inicial al estado deseado en el desarrollo de la personalidad y la formación de los educandos se condiciona con un sistema de acciones tendientes a obtener objetivos en los ámbitos personal y colectivo, como a nivel de conjunto de la institución educativa. (pág. 19); Dang, et al. (2018), en sus hallazgos sugieren que las tareas diseñadas para promover la metacognición pueden tener un impacto en los estudiantes y pueden proporcionar los mayores beneficios a los estudiantes de bajo rendimiento.

En cuanto a la variable; logros de aprendizaje, Mamani (2017), señala que viene a ser el resultado de todos los aprendizajes obtenidos por los educandos al finalizar su ejercicio académico como consecuencia del proceso de enseñanza y aprendizaje, que se logran medir con los indicadores de esos logros y que permite observar no sólo el desempeño del educando sino también analizar lo que está sucediendo, este análisis le puede llevar al docente a replantear sus estrategias, métodos y procedimientos de enseñanza para lograr los objetivos planteados, en otra palabras es el resultado medido cuantitativamente 
del aprendizaje, siendo que este autor indica que la evaluación de estos logros se interpreta como el proceso analítico y reflexión académica, que docente debe acatar y a partir de ello construir o rediseñar estrategias y el estudiante reflexionara sobre su aprendizaje; no sólo se debe medir los logros, sino también evaluarlos, no solo merecen una calificación sino que requieren de todo una valoración del proceso de enseñanza. El logro de resultados se puede medir por el nivel de conocimiento alcanzado, por el dominio y destreza en el área.

\section{ESTRATEGIAS METODOLÓGICAS O MATERIALES Y MÉTODOS}

El diseño es no experimental, correlacional, transversal, ya que busca establecer la relación de dos variables en un determinado momento sin manipular las variables en estudio dentro de un enfoque cuantitativo (Hernández et al. 1991).

Esquema del diseño:

\section{Leyenda:}

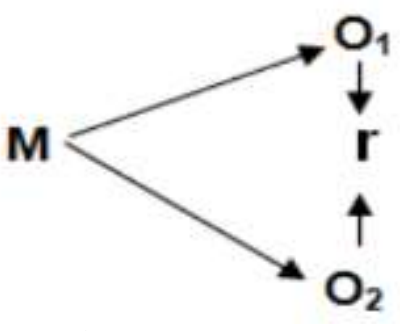

M= Muestra observada (estudiantes de la Institución Educativa N81014).

$r=$ Relación causal entre las variables estudiadas.

O1 = Observación de la variable relacional1: (Estrategias metacognitivas).

O2 = Observación de la variable relaciona 12: (Logros de aprendizaje).

La población objeto de investigación, estuvo compuesta de acuerdo a especificaciones comunes que presentan los estudiantes; conformada por 155 estudiantes varones y mujeres. (Gómez, 2006) 


\section{RESULTADOS Y DISCUSIÓN}

Tabla 1: Categorías de Estrategia Metacognitiva

\begin{tabular}{llcc}
\hline & Categorías & Frecuencia & Porcentaje \\
\hline Válido & Bajo & 0 & 0,0 \\
\cline { 2 - 4 } & Medio & 13 & 13,7 \\
\cline { 2 - 4 } & Alto & 82 & 86,3 \\
\cline { 2 - 4 } & Total & 95 & 100,0 \\
\hline
\end{tabular}

Nota: Data de estrategias metacognitivas

\section{Interpretación:}

En la tabla 1 se muestra que existe predominio en Estrategias metacognitivas de la categoría alto con $86,3 \%$ ( 82 estudiantes), en menor dominancia la categoría medio con 13,7 \% (13 estudiantes). A decir de Chamblé, (2020) concluye que la comprensión lectora tiene una relación directa con el uso de las estrategias metacognitivas, lo cual le permite realizar la interacción continua con los textos que lee, a partir del cual construye interpretaciones

Tabla 2: Categorías de Logros de aprendizaje en Personal social

\begin{tabular}{llccc}
\hline & & F & \% \\
\hline Válido & Bajo & 0 & 0,0 \\
\cline { 2 - 4 } & Medio & 93 & 97,8 \\
\cline { 2 - 4 } & Alto & 2 & 2,2 \\
\hline Total & 95 & 100,0 \\
\hline
\end{tabular}

Nota: Data de la variable logros de aprendizaje

\section{Interpretación:}

En la tabla 2, se muestra que existe predominio en Logros de aprendizaje de la categoría medio con $97,8 \%$ (93 estudiantes), en menor dominancia el nivel alto con 2,2 \% (2 estudiantes). Parte inferencial, con la prueba de normalidad según el tipo de distribución de datos usando, para la relación entre Estrategias Metacognitivas y Logros de aprendizaje en el área de Personal Social se empleó la fórmula de Rho de Spearman. Klimenko, (2009) hace hincapié que para la enseñanza de las estrategias cognitivas y metacognitivas es necesario que metodológicamente deben estar en concordancia con el currículo, con los recursos educativos, tener continuidad y ser conscientes de perfeccionamiento. 
Tabla 3: Coeficiente de correlación entre Estrategias metacognitivas y Logros de aprendizaje

\begin{tabular}{lllcc}
\hline & & Estrat. Metacog. & Logros de Apren. \\
\hline $\begin{array}{l}\text { Rho de } \\
\text { Spearman }\end{array}$ & $\begin{array}{l}\text { Estrategias } \\
\text { metacognitivas }\end{array}$ & $\begin{array}{l}\text { Coeficiente de } \\
\text { correlación }\end{array}$ & 1,000 &, $804^{* *}$ \\
\cline { 3 - 5 } & & Sig. (bilateral) & $\cdot$ &, 000 \\
\cline { 2 - 5 } & $\begin{array}{l}\text { Logros de } \\
\text { aprendizaje }\end{array}$ & $\begin{array}{l}\text { Coeficiente de } \\
\text { correlación }\end{array}$ &, $804^{* *}$ & 95 \\
\cline { 2 - 5 } & $\begin{array}{l}\text { Sig. (bilateral) } \\
\text { N }\end{array}$ &, 000 & 1,000 \\
\hline
\end{tabular}

\section{Interpretación:}

Nota: **. La correlación es significativa en el nivel 0,01 (bilateral).

La tabla 3, muestra el coeficiente de correlación de Spearman, a través de la cual se demuestra que existe una relación directa y fuerte entre estas dos variables, la misma que estadísticamente es altamente significativa $\left(\mathrm{Rho}=0,804^{* *}, \mathrm{p}=0.000<0.05\right)$ Por lo tanto, se rechaza la hipótesis nula (Ho); y se confirma la hipótesis (Ha) que dice que existe una relación directa y significativa entre las estrategias metacognitivas y el logro de aprendizajes en el área de personal social de los estudiantes del V ciclo de una institución educativa de Trujillo. Se corrobora los aportes de la teoría de Flavell (1985) por cuanto plantea que la habilidad metacognitiva regula cualquier actividad cognitiva propias en la tercera infancia y adolescencia.

Tabla 4: Prueba de normalidad de Estrategias metacognitivas y Logros de aprendizaje

\begin{tabular}{lcccc}
\hline & \multicolumn{3}{c}{ Kolmogorov-Smirnov $^{\mathbf{a}}$} \\
\hline ESTRAT META & Estadístico & gl & Sig. \\
\hline D 1 V! &, 191 & 95 &, 000 \\
\hline D 2 V1 &, 177 & 95 &, 000 \\
\hline D 3 V1 &, 166 & 95 &, 000 \\
\hline LOGROS DE APR &, 249 & 95 &, 000 \\
\hline CI &, 184 & 95 &, 000 \\
\hline CPD &, 106 & 95 &, 010 \\
\hline CIH &, 154 & 95 &, 000 \\
\hline GEA &, 164 & 95 &, 000 \\
\hline GRE &, 150 & 95 &, 000 \\
\hline
\end{tabular}

Nota: Data de Estrategias metacognitivas y Logros de aprendizaje 


\section{Interpretación:}

Visto los valores de Sig. de Estrategias metacognitivas y Logros de aprendizaje que, son $<0.05$, se precisa que la distribución de los datos es no paramétrica, por ello debe de usarse la fórmula de Rho Spearman para hallar las relaciones entre variables y dimensiones. Por lo tanto, se rechaza la hipótesis nula (Ho); y se confirma la hipótesis (Ha) que dice que existe una relación directa y significativa entre las estrategias metacognitivas y el logro de aprendizajes en el área de personal social de los estudiantes del V ciclo de una institución educativa de Trujillo. Se corrobora los aportes de la teoría de Flavell (1985) por cuanto plantea que la habilidad metacognitiva regula cualquier actividad cognitiva propias en la tercera infancia y adolescencia.

$\mathrm{Al}$ analizar los resultados de la prueba de hipótesis general se ha encontrado que existe relación $(\mathrm{p}=0,000)$ positiva y fuerte entre Estrategias metacognitivas y logros de aprendizaje (Rho Spearman $0.804 * *$ ) y una influencia de $77,5 \%$ tal como se presenta en las Tablas 3. A la luz de los resultados, se rechaza la Ho y se acepta la Ha que predice la existencia de una relación significativa entre ambas variables; por lo que se concluye reforzando tal afirmación.

Las afirmaciones que se derivan de los resultados concuerdan con los hallazgos de Martel y Verastegui, (2016) que al desarrollar su investigación doctoral denominado Relación entre estrategias metacognitivas y logro de competencias del área de personal social, concluyó que las estrategias metacognitivas son un conjunto de habilidades, capacidades y destrezas que permiten alcanzar los logros de aprendizaje, para lo cual el docente cumple un rol preponderante, puesto que debe facilitar su desarrollo. Así mismo Cansaya, (2018) afirma que cuando los estudiantes usan estrategias metacognitivas adquieren un nivel de conciencia sobre su propia forma de pensar, con el fin de modificarlas periódicamente en función de sus objetivos personales.

De igual manera, estos resultados permiten reafirmar los supuestos de la teoría del aprendizaje significativo de Bruner (citado en Dale, 1997) cuando considera que, los seres humanos construyen aprendizajes cuando interactúan en situaciones reales y guiados por la motivación y la curiosidad, situación que les permite hacer conexiones a nivel cognitivo teniendo como base sus conocimientos previos. Estos conocimientos previos están en relación a su experiencia y conocimiento con los que se enfrenta las situaciones retadoras. 
Sin embargo, existen otros factores que inciden en un $23,5 \%$ siendo los específicamente del ámbito pedagógico.

Las afirmaciones entorno a los resultados, están en concordancia con los estudios de Mejía, (2019) en cuyo estudio denominado La metacognición y el aprendizaje en estudiantes de Bachillerato del Ecuador, quien concluyó afirmando a partir de sus resultados, que al existir una relación significativa entre ambas variables, se precisa que la metacognición ayuda a fortalecer los procesos de autorreflexión a partir del análisis de los elementos usados para el aprendizaje, permitiendo la regulación recurrente de los mismos. En esa misma línea de ideas, Chamblé, (2020) afirma que la comprensión lectora tiene una relación directa con el uso de las estrategias metacognitivas, lo cual le permite realizar la interacción continua con los textos que lee, a partir del cual construye interpretaciones.

Las afirmaciones en relación a los resultados, están en concordancia con los estudios de Arteta y Huaire (2016) en cuyo estudio denominado Estrategias metacognitivas y concepciones de aprendizaje en estudiantes universitarios, concluye haciendo afirmaciones referentes a la existencia de la relación directa entre las variables en mención y sus dimensiones, explicando que, los logros de aprendizaje en general, se alcanzan en la medida que se tenga claro el camino a seguir y a donde llegar. En tal sentido, a decir de Córdova y Marroquín (2018); Cabero et al, (2017) coinciden en sostener que el logro de los aprendizajes se gestiona de forma autónoma, a partir de la metacognición.

Por otro lado, desde la teoría sociocultural de Vygotsky (como se citó en Dale, 1997) se sustenta la construcción de aprendizajes en un contexto socio cultural, es decir el individuo recibe la mediación del docente así como de los factores culturales, sociales, familiares, etc. los mismos que son incorporados en los proceso de aprendizaje mediante niveles, denominados zonas de desarrollo (ZD), así mismo desde la teoría de los procesos metacognitivos, se refiere que el individuo regula cualquier procesos cognitivo, con el fin de lograr aprendizajes; sin embargo, existen otros factores que influyen en estos procesos en un $59,8 \%$.

Los resultados, concuerdan con los hallazgos de Chia et al, (2019) en cuyo estudio denominado Desarrollo de estrategias metacognitivas para fortalecer la metacognición en estudiantes, concluyó afirmando que la conciencia metacognitiva se mejoró a partir del desarrollo de estrategias metacognitivas propuestas en el desarrollo de las actividades de 
aprendizaje de los estudiantes dentro de sus aulas. En tal sentido y en concordancia también con los aportes de Osses y Jaramillo (2008) se precisa que la metacognición permite a los niños a desarrollar la capacidad de gestión de sus aprendizajes de manera autónoma.

Los resultados, concuerdan con los hallazgos de Puma, (2020) cuando en su estudio denominado Relación de estrategias metacognitivas y el desarrollo del rendimiento académico en estudiantes universitarios, concluyendo que el rendimiento académico es relativo a las estrategias que los estudiantes utilizan para regular la cognición, motivo por la cual sugiere incorporar más a menudo este tipo de estrategias en el desarrollo de proceso pedagógicos de los docentes de todos los niveles educativos. En esa misma línea de ideas, Laguna y Trujillo, (2017) señalan que los estudiantes que utilizan diversas estrategias metacognitivas, resaltan características comunes como: la formulación de preguntas apropiadas, mejor planificación de actividades, gestión y control de todos los procesos

\section{CONCLUSIÓN O CONSIDERACIONES FINALES}

Se concluye que existe relación $(p=0,000)$ positiva y fuerte entre Estrategias metacognitivas y logros de aprendizaje (Rho Spearman 0.804**) y una influencia de 77,5 $\%$. También, existe relación $(\mathrm{p}=0,000)$ positiva y moderada entre estrategias metacognitivas y construye su identidad (Rho Spearman $0.487 * *$ ) con una influencia del $26,4 \%$. De igual forma, existe relación $(\mathrm{p}=0,000)$ positiva y moderada entre estrategias metacognitivas y convive y participa democráticamente (Rho Spearman $0.657 * *$ ) con una influencia del 52,6 \%. Se evidencia que, existe relación $(\mathrm{p}=0,000)$ positiva y moderada entre Estrategias metacognitivas y Construye interpretaciones históricas (Rho Spearman $0.692^{* *}$ ) con una influencia del 49,8\%. Como también, existe relación ( $\left.\mathrm{p}=0,000\right)$ positiva y moderada entre Estrategias metacognitivas y gestiona el espacio y el ambiente (Rho Spearman $0.544 * *)$ con una influencia del $40,2 \%$ y la existencia de la relación $(\mathrm{p}=0,000)$ positiva y moderada entre estrategias metacognitivas y gestiona los recursos económicos (Rho Spearman $0.594 * *$ ) con una influencia del 39,0\%

Finalmente se concluye que, existe relación $(\mathrm{p}=0,000)$ positiva y fuerte entre Logros de aprendizaje y la dimensión pedagógica (Rho Spearman $0.759 * *$ ) con una influencia del $66,2 \%$ De igual forma, existe relación $(\mathrm{p}=0,000)$ positiva y fuerte entre Logros de aprendizaje y la dimensión metodológica (Rho Spearman $0.704^{* *}$ ) con una influencia del 
$65,5 \%$. y también, existe relación $(\mathrm{p}=0,000)$ positiva y fuerte entre Logros de aprendizaje y la dimensión didáctica (Rho Spearman $0.742 * *$ ) con una influencia del 63,6 \%

\section{LISTA DE REFERENCIAS}

Ajaja, P. (2017). Collection and analysis of students' metacognitive orientations for science learning: A survey of science classrooms in Delta State, Nigeria. Electronic Journal of Science Education.

Alvarado, J. y Lunarejo, D. (2017). Estrategias cognitivas de aprendizaje y el nivel de razonamiento geométrico en los estudiantes, especialidad de Mecánica de Producción I.E.S.T.P. Manuel Seoane Corrales. Lima - 2015. Tesis de maestría. Universidad Cesar Vallejo. Lima, Perú. Obtenido de https://repositorio.ucv.edu.pe/bitstream/handle/20.500.12692/5071/Alvarado_E J-Lunarejo_LDJ.pdf?sequence $=1$

Anaya, E. (2018). “Aplicación de estrategias cognitivas y metacognitivas y su influencia en la comprensión lectora en primaria Mirones Lima - 2017”. Tesis de grado. Universidad Nacional Federico Villarreal. Lima, Perú. Obtenido de http://repositorio.unfv.edu.pe/bitstream/handle/UNFV/2362/Anaya\%20Castro $\%$ 20Evila\%20Amanda.pdf? sequence $=1 \&$ is Allowed $=\mathrm{y}$

Cabero Almenara, J., Piñoro Virué, R., \& Reyes Rebolledo, M. M. (2017). Material educativo multimedia para el aumento de estrategias metacognitivas de comprensión lectora. España: Universidad de Sevilla.

Cansaya, V. (2018). Las estrategias metacognitivas y el aprendizaje significativo en el área de Arte, Colegio Emblemático del Perú "Mateo Pumacahua”, Cuzco, año 2015. Tesis de maestría. Universidad Nacional de Educación Enrique Guzmán Valle. Lima, Perú. Obtenido de https://repositorio.une.edu.pe/bitstream/handle/UNE/1946/TM\%20CE-Pse\%203659\%20C1\%20$\% 20$ Cansaya \%20Aquino.pdf?sequence $=1 \&$ is Allowed $=\mathrm{y}$

Chablé Tolosa, M. (2020). La comprensión lectora y su relación con el uso de estrategias metacognitivas de lectura en universitarios. Revista Internacional de Estudios en Educación. México: Instituto Valle Dorado. 
Chia Chen, T., Chih Ming, C., \& Hahn Ming, L. (2019). Effectiveness of a digital penbased learning system with a reward mechanism to improve learners' metacognitive strategies in listening. Computer Assisted Language Learning.

Córdova Urbano, D. L., \& Marroquín Yerovi, M. (2018). Mejoramiento del rendimiento académico con la aplicación de estrategias metacognitivas para el aprendizaje significativo. Colombia: Universidad Mariana, San Juan de Pasto.

Dang, N. V., Chiang, J. C., Brown, H. M., \& McDonald, K. K. (2018). Curricular Activities that Promote Metacognitive Skills Impact Lower-Performing Students in an Introductory Biology Course. JOURNAL OF

\section{MICROBIOLOGY \& BIOLOGY EDUCATION.}

Gómez, M. (2006). Introducción a la metodología de la investigación. Argentina: Brujas. Obtenido de https://books.google.com.pe/books?id=9UDXPe4U7aMC\&dq=Metodolog\%C3 $\% \mathrm{ADa}+\mathrm{de}+\mathrm{la}+$ investigaci\%C3\%B3n:la+poblacion\&source=gbs_navlinks_s

Gonzales, J. (2013). Los niveles de conocimiento. El Aleph en la innovación curricular. Scielo. Obtenido de http://www.scielo.org.mx/scielo.php?script=sci_arttext\&pid=S166526732014000200009

Hernández, R.; Fernández, C. y Baptista, M. (2014). Metodología de la investigación. México: McGRAW-HILL / INTERAMERICANA EDITORES, S.A. DE C.V. Obtenido de https://www.esup.edu.pe/descargas/dep_investigacion/Metodologia\%20de\%20 la\%20investigaci\%C3\%B3n\%205ta\%20Edici\%C3\%B3n.pdf

Jaramillo, L. y Simbaña, V. (2014). LA METACOGNICIÓN Y SU APLICACIÓN EN HERRAMIENTAS VIRTUALES DESDE LA. Redalyc. Obtenido de https://www.redalyc.org/pdf/4418/441846097014.pdf

Laguna, S. y Trujillo, E. (2017). Efecto de las estrategias cognitivas y metacognitivas de lectura en la comprensión lectora de los estudiantes de cuarto grado de primaria de la Institución Educativa Silvia Ruff de Huari, 2014. Tesis de maestría. Universidad Católica Sedes Sapientiae. Lima, Perú. Obtenido de http://repositorio.ucss.edu.pe/bitstream/handle/UCSS/562/Laguna_Trujillo_tesi s_maestria_2017.pdf?sequence=1\&isAllowed $=y$ 
Lestari, W., \& Jailani. (2018). Enhancing an Ability Mathematical Reasoning through Metacognitive Strategies. Journal of Physis: Conference Series.

Mamani, G. (2017). Estrategias de enseñanzas y logro de aprendizaje en el área de historia, geografía y economía de los estudiantes del tercer grado de la institución educativa secundaria " Carlos Rubina Burgos". Tesis de grado. Universidad Nacional del Altiplano. Puno, Perú. Obtenido de http://tesis.unap.edu.pe/bitstream/handle/UNAP/5371/Mamani_Huanacuni_Gl oria_Yovanna.pdf?sequence $=1 \&$ isAllowed $=y$

Martel, G. y Verastegui, E. (2016). "Relación entre estrategias metacognitivas y logro de competencias del área de personal social en alumnos de la Institución Educativa 34116 de Yanacocha - Yanahuanca - Daniel Carrión”. Tesis de grado. Universidad Nacional Daniel Alcides Carrión". Cerro de Pasco, Perú. Obtenido de http://repositorio.undac.edu.pe/bitstream/undac/279/1/T026_70089503_T.pdf

MINEDU. (2018). Obtenido de http://umc.minedu.gob.pe/resultados-ece-2018/

Muhid, A., Eka Rizki, A., Hilaliyah, H., Budiana, N., \& Nizarudin Wagdi, M. (2020). The Effect of Metacognitive Strategies Implementation on Students' Reading Comprehension Achievement. International Journal of Instruction, 847-862.

Osses, S. y Jaramillo, S. (2008). METACOGNICION: Un camino para aprender a aprender. Scielo. Obtenido de https://scielo.conicyt.cl/pdf/estped/v34n1/art11.pdf

Puma Camargo, M. I. (2020). Relación de las estrategias metacognitivas y le desarrollo del rendimiento académico en estudiantes de la Escuela Profesional de Educación de la Universidad Nacional Amazónica Madre de Dios, 2017. Tesis de grado. Universidad Nacional Mayor de San Marcos. Lima.

Rogers, J. (2018). Teaching/Developing Vocabulary Through Metacognition. The TESOL Encyclopedia of English Language Teaching, First Edition. .

Sánchez, M.; García, J.; Steffens, E. y Hernández H. (2017). Estrategias Pedagógicas en Procesos de Enseñanza y Aprendizaje en la Educación Superior incluyendo Tecnologías de la Información y las Comunicaciones. Scielo. Obtenido de https://scielo.conicyt.cl/scielo.php?script=sci_arttext\&pid=S071807642019000300277 
Sierra, R. (2007). La estrategia pedagógica. Varona. Obtenido de https://www.redalyc.org/pdf/3606/360635565004.pdf

Solórzano Restrepo, J., \& López Vargas, O. (2019). Differential effect of a metacognitive scaffolding in a e-Learning environment over cognitive load, learning achievement ad metacognitive consciousness. Suma Psicológica, 3745.

Susantini, E., Bambang Sumitro, S., Duran Corebima, A., \& Susilo, H. (2018). Improving learning process in genetics classroom by using metacognitive strategy. Asia Pacific Education Review. 